\title{
Identification of Genetic Loci of Black Point in Chinese Common Wheat by Genome-Wide Association Study and Linkage Mapping
}

\author{
Guoguo Lv, ${ }^{1}$ Zhongdong Dong, ${ }^{1}$ Yudan Wang, ${ }^{1}$ Junyou Geng, ${ }^{1}$ Jia Li, ${ }^{1}$ Xialei Lv, ${ }^{1}$ Congwei Sun, ${ }^{1}$ Yan Ren, ${ }^{1}$ Jianwei Zhang, \\ and Feng Chen ${ }^{1, \dagger}$ \\ ${ }^{1}$ National Key Laboratory of Wheat and Maize Crop Science/Agronomy College, Henan Agricultural University, Zhengzhou, \\ Henan 450046, China \\ ${ }^{2}$ Henan Key Laboratory of Nuclear Agricultural Sciences/Isotope Institute Co., Ltd, Henan Academy of Sciences, Zhengzhou, \\ Henan 450015, China
}

\begin{abstract}
Black point is a common disease in wheat all over the world. The disease could downgrade wheat quality and cause human health problems. In this study, 406 wheat cultivars were used to investigate black point resistance. In the field tests, $20,65.5$, and $14.5 \%$ of the tested cultivars were resistant, moderately resistant, and susceptible, respectively, suggesting that improving black point resistance is necessary in Chinese wheat breeding. A genome-wide association study (GWAS) identified 386 single-nucleotide polymorphisms (SNPs) significantly related to black

three quantitative trait loci (QTL) for black point resistance- $Q B P$. hau3A, QBP.hau-6D, and QBP.hau-7D - with 6.76, 7.79, and 8.84\% phenotypic variation explained, respectively. Based on both the GWAS and linkage analyses, QBP.hau- $6 D$ covered six significant SNPs from the GWAS, and the position of these SNPs indicated that this QTL is a new locus for black point resistance. This study provides valuable germplasm for breeding wheat cultivars with resistance to black point and information for further understanding of molecular and genetic basis of black point resistance.
\end{abstract} point resistance in the tested wheat cultivars, and they were located on all chromosomes. Linkage mapping in a biparental population identified
Keywords: common wheat, black point, GWAS, linkage mapping
Black point is one of the important common diseases in wheat all over the world (Sissons et al. 2010). The obvious symptom of black point is dark-brown to black discoloration of the embryo end of wheat kernels, and it can cover most of the surface of the kernels (Conner and Davidson 1988; Fernandez and Conner 2011). The disease can lead to grain quality and value losses (Rees et al. 1984). Black point could influence many traits of wheat such as falling number, dough stability, germination, seed emergence, plant vigor, and plant growth, and even causes seedling blight (Cromey and Mulholland 1988; Dexter and Matsuo 1982; Fernandez et al. 2014; Hudec 2007). Previous reports showed that black point was associated with fumonisin contamination and might cause esophageal cancer and neural-tube defects in some human populations (Busman et al. 2012; Desjardins et al. 2007; Marasas 2001; Palacios et al. 2015).

Some studies showed that black point was mainly caused by abiotic stresses and high humidity, and extreme temperatures during grain filling easily lead to wheat black point (Fernandez et al. 2014; Maloy and Specht 1988). However, the cause of black point is still unclear and controversial. In most studies, fungi are proved as the main causal agent of black point; for example, Alternaria alternata (Busman et al. 2012; Perelló et al. 2008; Somma et al. 2019; Southwell et al. 1980), Fusarium proliferatum (Busman et al. 2012; Desjardins et al. 2007), and Bipolaris sorokiniana (Kumar

${ }^{\dagger}$ Corresponding author: F. Chen; chf0088@163.com

Funding: This work was funded by the National Key Research and Development Program (2016YFD0100502), the National Natural Science Foundation of China (U1804234), Henan Major Science and Technology Projects (181100110200-0102), and The Ten Thousands Plan (Z04295) of China.

*The $\boldsymbol{e}$-Xtra logo stands for "electronic extra" and indicates that there are supplementary materials published online.

The author(s) declare no conflict of interest.

Accepted for publication 10 February 2020.

(C) 2020 The American Phytopathological Society et al. 2002). However, a single fungal species may not be the only cause of black point. For example, the presence of A. alternata in both susceptible and resistant wheat cultivars was observed (Hudec 2007; Williamson 1997), indicating no direct relationship between fungal infection and black point. The formation of black point may be caused by enzymatic browning after suffering from stress such as polyphenol oxidase (Fuerst et al. 2014), peroxidases (POD) (Fernandez et al. 2014; March et al. 2007), and lipoxygenase (Porta and Rocha-Sosa 2002). These enzymes catalyze oxidation of phenolic compounds to brown or black pigments (melanins and quinines) (Fuerst et al. 2014; Tomás-Barberán and Espín 2001). Black point may be triggered by high humidity, extremes of temperature, and pregermination during the late stages of grain filling and ripening. Some cultivars showing susceptibility to black point possess relatively high POD and phenylalanine ammonia-lyase, an enzyme involved in phenolic acid biosynthesis activities (March et al. 2007).

There are some strategies to control black point such as cultural, biological, and chemical control but the most effective, economical, and environmentally sustainable way is to plant resistant cultivars (El-Gremi et al. 2017; Lehmensiek et al. 2004; Li et al. 2014; Liu et al. 2016). The development of black point mainly depends on environmental conditions, and severity is mostly influenced by wheat genotype (Fernandez and Conner 2011; Lehmensiek et al. 2004). The incidence of black point ranges from 0.3 to $66.7 \%$ (El-Gremi et al. 2017; Ellis et al. 1996; Li et al. 2014; Walker et al. 2008), and the heritability of black point is approximately $50 \%$ ( $\mathrm{Li}$ et al. 2014).

Studies on the genetic basis of black point resistance have commonly involved traditional linkage-mapping methods using biparental populations (Lehmensiek et al. 2004; March et al. 2007). Lehmensiek et al. (2004) used low-density simple-sequence repeat (SSR) markers and identified black point-resistant quantitative trait loci (QTL) on chromosomes 1D, 2B, 3D, 4A, 5A, and 7A in the population Sunco/Tasman, and on $2 \mathrm{~A}, 2 \mathrm{D}$, and $7 \mathrm{~A}$ in the population Cascades/AUS1408, with phenotypic variation explained (PVE) values of 4 to $15 \%$ and 12 to $18 \%$, respectively. Liu et al. (2016) mapped 9 QTL on chromosomes 2AL, 2BL, 3AL, 3BL, 5AS, 6A, 7AL (2), and 7BS in a recombinant inbred line (RIL) population, and found 25 loci on chromosomes 2A, 2B, 3A, 3B (2), 3D, 4B (2), 5A (3), 
5B (3), 6A, 6B, 6D, 7A (5), 7B, and 7D (2) (Liu et al. 2017), with PVE values of 7.9 to $18.0 \%$, using the genome-wide association study (GWAS) approach.

With the rapid development of sequencing techniques, highdensity single-nucleotide polymorphism (SNP) markers have been widely used to detect new genes or QTL in common wheat through GWAS and linkage mapping for complex traits. Black point is becoming one of the important diseases in the Huanghuai Valley, the most important wheat region in China (with 60 to $70 \%$ of the total wheat production area). This study aims to screen wheat germplasm resistant to black point and identify novel resistance loci for markerassisted selection as well as provide useful information for further understanding of the molecular and genetic basis of black point resistance in common wheat.

\section{Materials and Methods}

Plant material. Two wheat panels were used in this study. Panel I consisted of 163 common wheat cultivars from Henan, Hebei, Shaanxi, Shanxi, and Shandong Provinces, and they were selected based on their pedigree, released region, agronomic performance, importance in breeding, and cultivated area (Chen et al. 2019; Sun et al. 2017). Panel II consisted of 243 common wheat cultivars or advanced breeding lines released or developed after 2014, representing
Table 1. Representative wheat accessions with different levels of resistance to black point disease

\begin{tabular}{lc}
\hline Disease incidence (\%) & Cultivars \\
\hline $0.1-4.9$ & Yanyou 361, Zhengzhou 8761, Linkang \\
& 5027, Xinong 6028, Yumai 8, Shannong \\
& 7859, Chinese spring, Xingyu 7, \\
Zhongfengmai 2, Xuke 877, Xinyanmai 98, & Xiaoyan 6, Xinong 979, Jinmai 49, Longmai \\
& 157, Neixiang 184, Aifeng 3, Zhongnong \\
& 28, Jinmai 47 \\
& Xinyin 13-2, Lumai 14, Beinongda 92, Jinmai \\
& 33, Yuanfeng 898, Wenmai 29, Xuke 158, \\
& Zhongmai 10, Lunxuan 167, Xiza 5, \\
& Shunmai 8, Yunong 805, Hemai 6, Hangmai \\
& 6, Jimai 26, Lumai 15, Huayan 328, Jiamai \\
$5-14.9$ & 329, Zhongle 8 \\
& Yanzhan 4110, Han 97-5297, Yumai 3, \\
& Pindong 34, Yumai 57, Xinmai 3306, \\
& Wenyu 019, Aomai 18, Luyuan 502, Tianle \\
& 6, Yumai 58, Xinmai 8, Yuanxie 62, Huabei \\
& 187, Caizhi 204, Zhengmai 9694, Zhoumai \\
23, Shunmai 299, Han 4589, Ningnong 718, \\
Taixue 30 \\
Yan 893, Xuan, Yimai 8
\end{tabular}

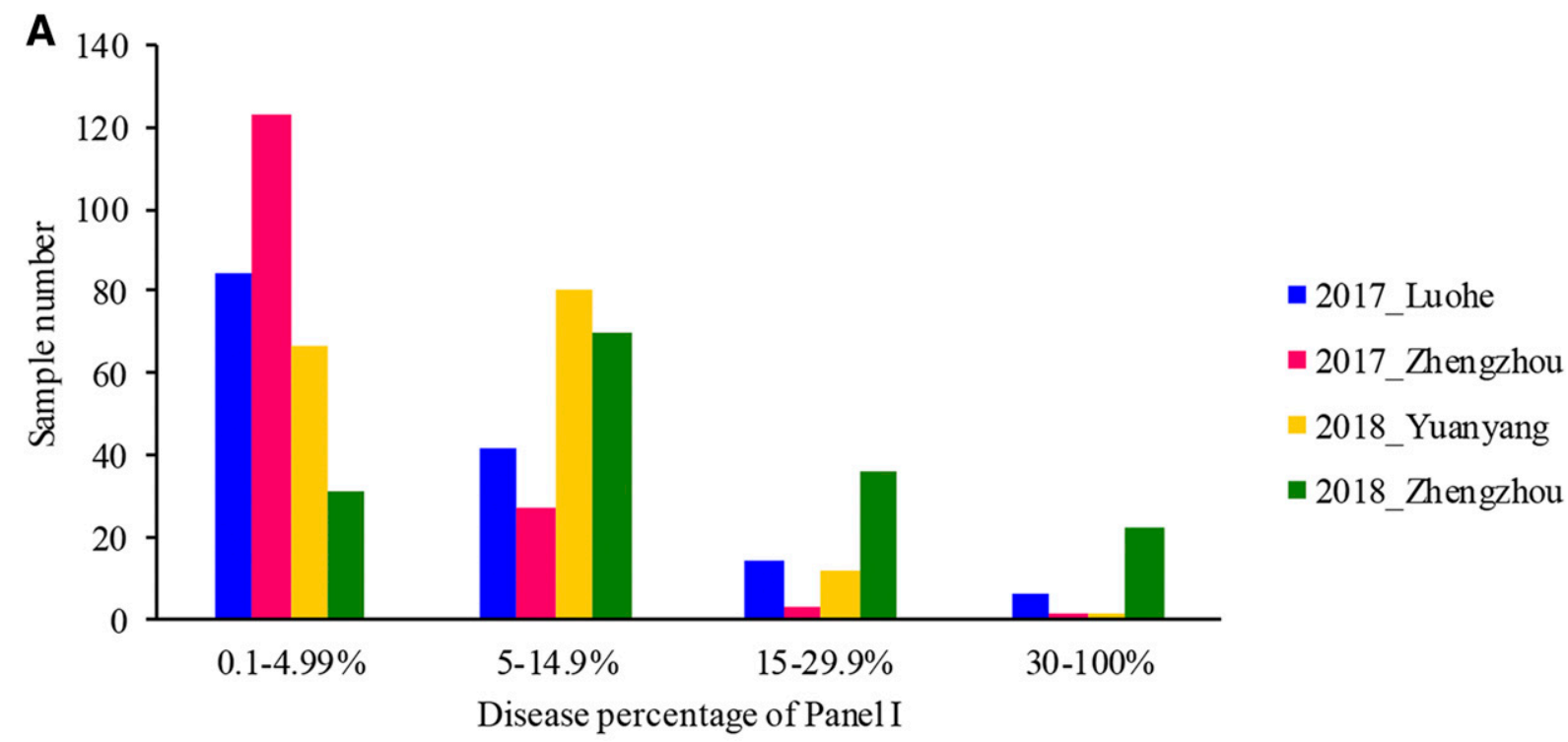

B

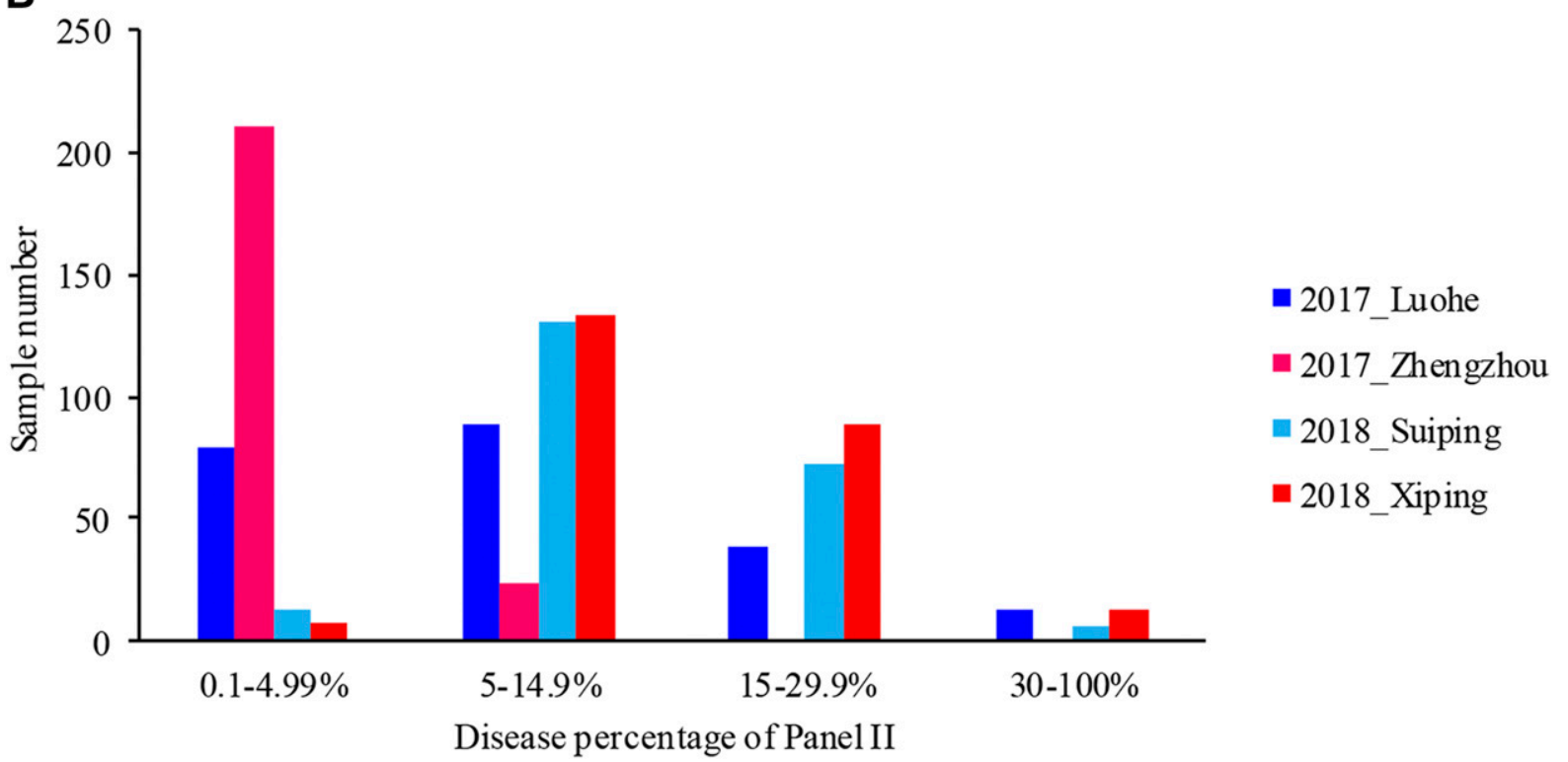

Fig. 1. Distribution of black point index in Chinese wheat cultivars in Panel I (A) and Panel II (B) in four environments. 
current breeding situations in the Huanghuai Valley (Yang et al. 2019). In 2017, Panels I and II were planted at the Scientific Research and Education Center (SREC) of Henan Agricultural University, in Luohe, and in Zhengzhou, with two replications at each location. In 2018, panel I was planted in Zhengzhou and Yuanyang, with two replicates at each location, and panel II was planted in Xiping and Suiping.

An $\mathrm{F}_{10}$ recombinant inbred line (RIL) population with 187 lines derived from a cross between UC1110 and PI610750 (UP) was used to map QTL for black point resistance. The RIL population was planted in the Zhengzhou SREC in 2017. All of the wheat accessions were performed using a completely randomized design. Each plot contained $122-\mathrm{m}$-long rows with $23 \mathrm{~cm}$ between neighboring rows and $10 \mathrm{~cm}$ between neighboring plants.

Phenotyping. After harvest and threshing, 100 grains of each accession were randomly selected from a well-mixed sample for phenotyping black point with three replications. Kernels with black point were calculated as the ratio of black point kernel number to the total kernel number. The mean value of black point in two replicates from
A

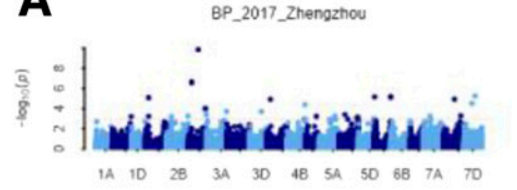

cromosome

BP_2017_Luone
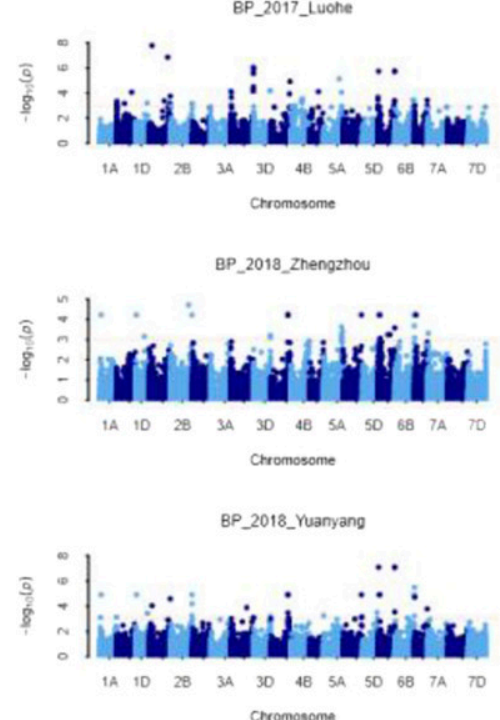

crromosome
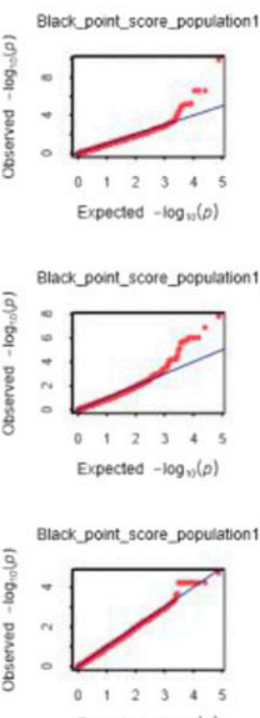

Expected $-\log _{n}(\rho)$

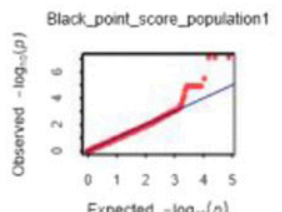

Expected $-\log _{n}(0)$
B

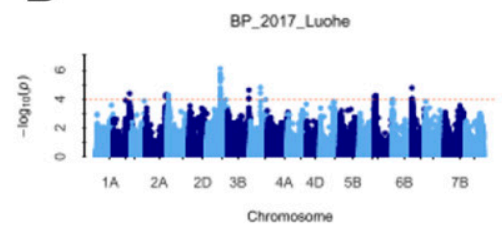

BP_2017_Zhengzhou

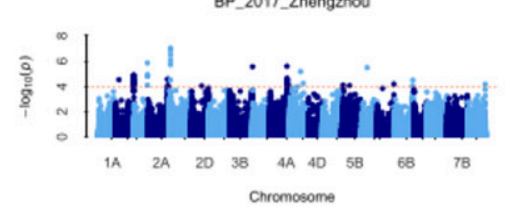

BP_2018_Xiping

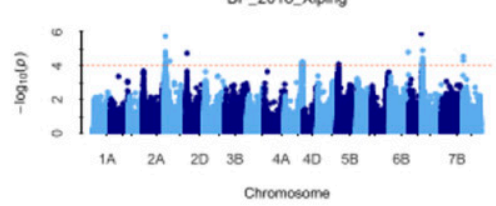

PP_2018_Suiping
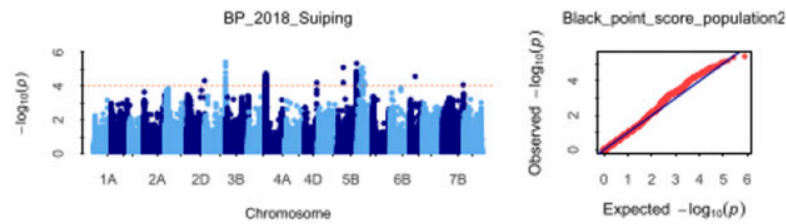

C

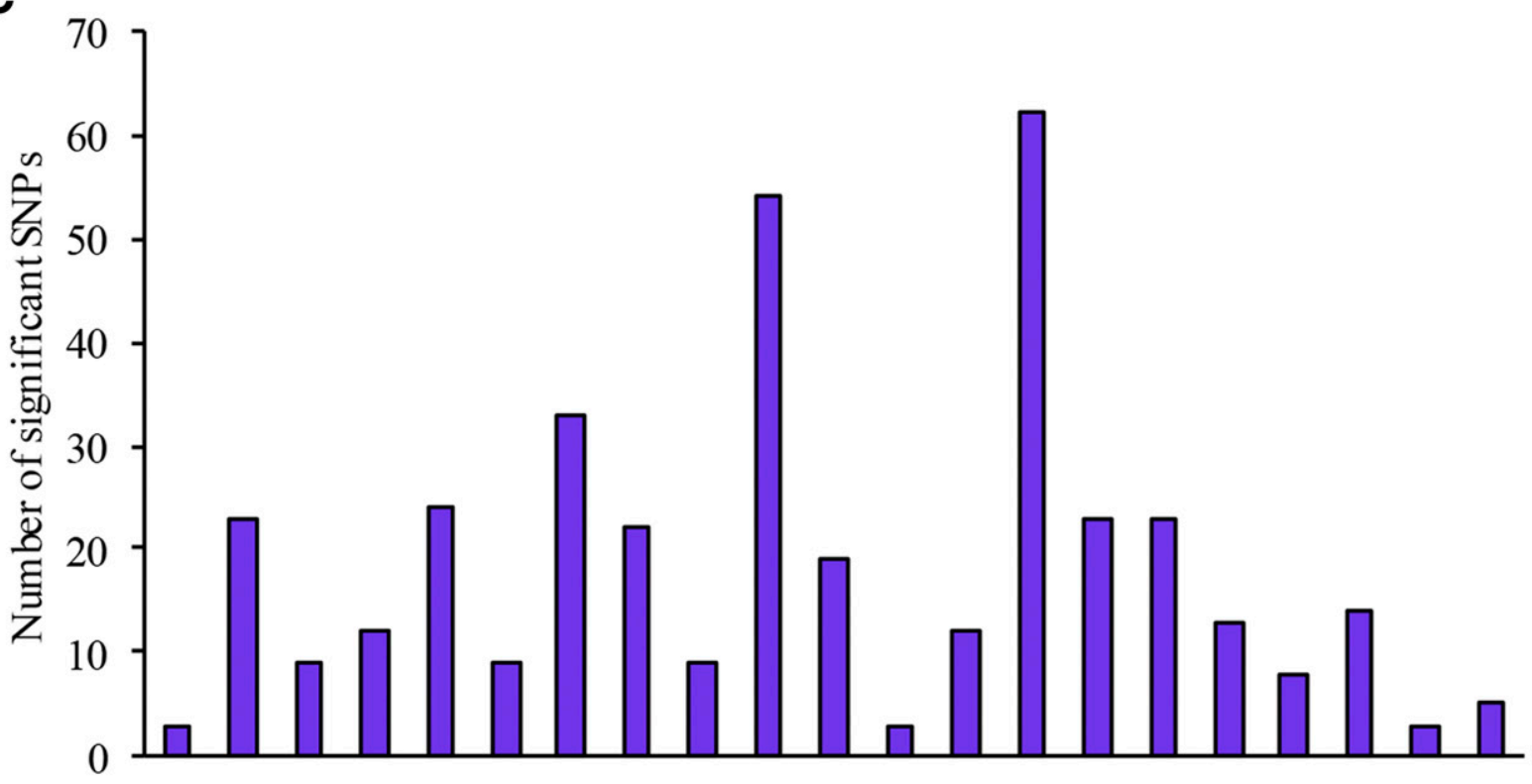

1A 1B 1D 2A 2B 2D 3A 3B 3D 4A 4B 4D 5A 5B 5D 6A 6B 6D 7A 7B 7D

\section{Chromosome}

Fig. 2. Manhattan and Q-Q plots for black point identified by genome-wide association study (GWAS) in A, panel I and B, panel II; and C, distribution of significant single-nucleotide polymorphisms (SNPs) on different chromosomes. 
Table 2. Multienvironmentally significant single-nucleotide polymorphisms (SNPs) identified by genome-wide association study in this study

\begin{tabular}{|c|c|c|c|c|}
\hline Year, location, SNP & $\mathrm{Chr}^{\mathbf{a}}$ & Position (bp) & $P$ value & $R^{2}(\%)^{\mathbf{b}}$ \\
\hline \multicolumn{5}{|c|}{ 2017_Luohe, 2017_Zhengzhou, 2018_Yuanyang } \\
\hline RAC875_rep_c71149_738 & $2 \mathrm{~B}$ & $782,536,180$ & 0.00052581 & 12.29 \\
\hline Excalibur_c10055_221 & $6 \mathrm{~A}$ & $28,017,180$ & $6.87 \mathrm{E}-06$ & 18.20 \\
\hline GENE-4167_145 & $6 \mathrm{~A}$ & $611,328,876$ & $6.87 \mathrm{E}-06$ & 18.20 \\
\hline BS00099401_51 & $6 \mathrm{~A}$ & $613,256,522$ & $6.87 \mathrm{E}-06$ & 18.20 \\
\hline \multicolumn{5}{|l|}{ 2017_Luohe, 2018_Yuanyang } \\
\hline GENE-1337_298 & $2 \mathrm{~A}$ & $107,383,862$ & $9.11 \mathrm{E}-05$ & 16.21 \\
\hline Kukri_c17_1587 & $2 \mathrm{~A}$ & $774,850,845$ & $2.63 \mathrm{E}-05$ & 17.80 \\
\hline BS00097147_51 & $2 \mathrm{~B}$ & $786,033,209$ & $6.29 \mathrm{E}-05$ & 16.68 \\
\hline Tdurum_contig51605_1130 & 3B & $822,680,599$ & 0.00085425 & 13.44 \\
\hline Tdurum_contig51605_194 & 3B & $822,682,075$ & 0.00085425 & 13.44 \\
\hline BS00087695_51 & $3 B$ & $822,826,547$ & 0.00085425 & 13.44 \\
\hline Tdurum_contig11114_335 & 3B & $822,828,808$ & 0.00085425 & 13.44 \\
\hline Tdurum_contig50364_1051 & $6 \mathrm{~B}$ & $711,702,135$ & 0.00058243 & 13.91 \\
\hline GENE-4021_496 & $6 \mathrm{D}$ & $463,743,572$ & 0.00016166 & 15.49 \\
\hline \multicolumn{5}{|l|}{ 2018_Yuanyang, 2018_Zengzhou } \\
\hline Excalibur_rep_c77404_218 & $1 \mathrm{~A}$ & $33,205,346$ & $1.24 \mathrm{E}-05$ & 18.78 \\
\hline D_contig25392_201 & $1 \mathrm{D}$ & $31,425,108$ & $1.24 \mathrm{E}-05$ & 18.78 \\
\hline RAC875_rep_c71149_798 & 2B & $782,536,120$ & $1.24 \mathrm{E}-05$ & 18.78 \\
\hline Kukri_c3831_2136 & $4 \mathrm{~A}$ & $634,841,828$ & $1.24 \mathrm{E}-05$ & 18.78 \\
\hline Kukri_c22504_459 & $4 \mathrm{~A}$ & $636,574,055$ & $1.24 \mathrm{E}-05$ & 18.78 \\
\hline Kukri_c2757_1535 & $4 \mathrm{~A}$ & $638,174,291$ & $1.24 \mathrm{E}-05$ & 18.78 \\
\hline Excalibur_rep_c102533_116 & $5 B$ & $677,154,676$ & $1.24 \mathrm{E}-05$ & 18.78 \\
\hline wsnp_CAP11_c3570_1730776 & $6 \mathrm{~A}$ & $61,409,322$ & $1.24 \mathrm{E}-05$ & 18.78 \\
\hline CAP7_c9162_265 & $6 \mathrm{~A}$ & $61,409,514$ & $1.24 \mathrm{E}-05$ & 18.78 \\
\hline Excalibur_c35871_596 & $6 \mathrm{~A}$ & $615,467,141$ & 0.00028336 & 14.79 \\
\hline Tdurum_contig25789_260 & $6 \mathrm{~B}$ & $702,751,203$ & $1.24 \mathrm{E}-05$ & 18.78 \\
\hline RAC875_c50572_106 & $6 \mathrm{~B}$ & $720,474,930$ & $3.24 \mathrm{E}-06$ & 20.55 \\
\hline RAC875_c57219_1058 & $6 \mathrm{~B}$ & $720,507,929$ & $1.24 \mathrm{E}-05$ & 18.78 \\
\hline \multicolumn{5}{|l|}{ 2017_Luohe, 2017_Zhengzhou } \\
\hline AX-109939573 & $2 \mathrm{~A}$ & $733,030,778$ & $8.36 \mathrm{E}-05$ & 18.54 \\
\hline
\end{tabular}

a Chromosome.

${ }^{\mathrm{b}} R^{2}$ of model with SNP.

A

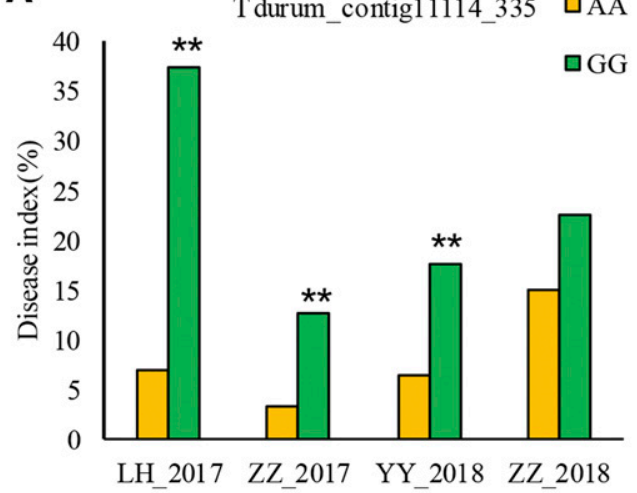

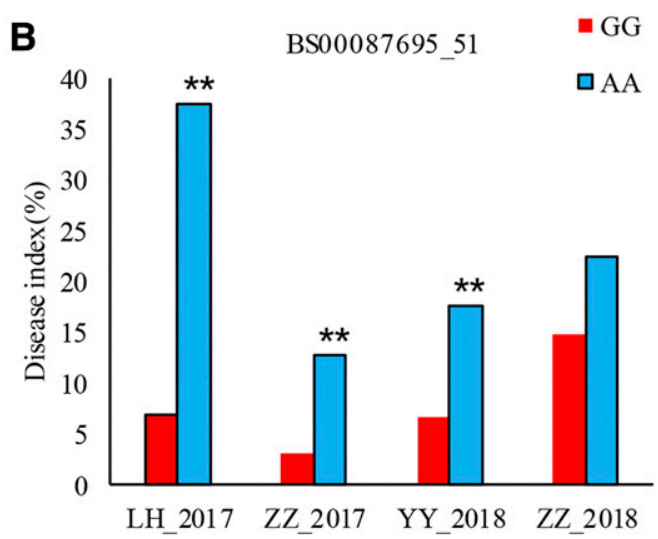
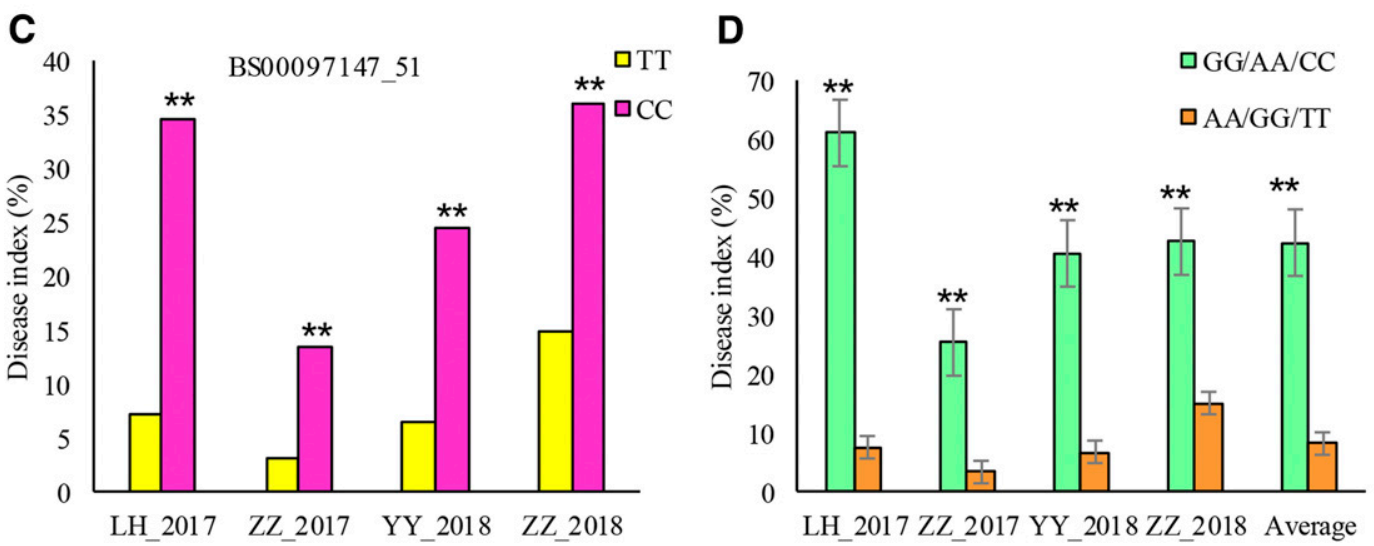

Fig. 3. Comparison of black point between cultivars with superior and inferior alleles in four environments: A, Tdurum_contig11114_335; B, BS00087695_51; C, BS00097147_51: and D, Combination of Tdurum_contig11114_335, BS00087695_51, and BS00097147_51. Asterisks $\left.{ }^{* *}\right)$ indicate $P<0.01$. 
the same environment was used for further analysis. Best linear unbiased prediction (BLUP) values were calculated across environments using the 'Ime4' package in $\mathrm{R}$ software, version 3.5.2. Function 'lmer' was used to fit a linear mixed-effects model. Afterward, function 'ranef' was used to get the random effect of BLUP. The final adjusted phenotype values were obtained by adding the random effect to averaged phenotype values. Based on a previous report (Kang et al. 1999), wheat with a black point percentage of 0.1 to $4.9 \%$ was regarded as resistant, 5 to $14.9 \%$ as moderately resistant,
15 to $29.9 \%$ as moderately susceptible, and greater than $30 \%$ as susceptible.

Genotyping and GWAS. Panels I and II were genotyped using the wheat $90 \mathrm{~K}$ and $660 \mathrm{~K}$ SNP arrays, respectively, as previously described (Chen et al. 2019; Sun et al. 2017; Yang et al. 2019). The quality pretreatment of genotyping data was carried out for SNP call rate and minor allele frequency with the PLINK software using the thresholds of maf 0.02 and geno 0.1 (http://zzz.bwh.harvard.edu/plink/tutorial. shtml) (Purcell et al. 2007). Population structure was assessed using

A
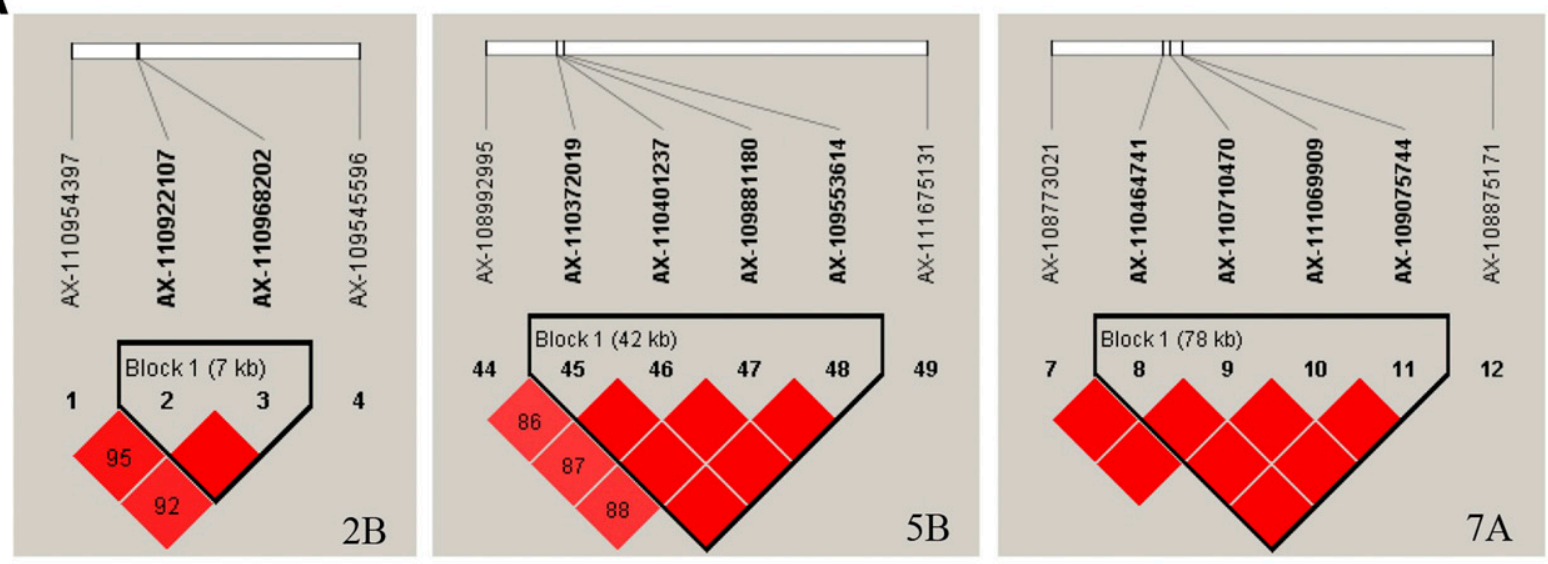

B

\begin{tabular}{|c|c|c|c|}
\hline Chromosome & Haplotype & Alleles & Black point (\%) \\
\hline \multirow{2}{*}{ 2B } & $2 B \_h a p_{-} l a$ & CG & 9.99 \\
\cline { 2 - 4 } & $2 B \_h a p_{-} l b$ & T A & $16.72^{* *}$ \\
\hline \multirow{2}{*}{ 5B } & $5 B \_h a p_{-} l a$ & GCGG & 10.16 \\
\cline { 2 - 4 } & $5 B \_h a p_{-} l b$ & CT AA & $12.97^{*}$ \\
\hline \multirow{2}{*}{ 7A } & $7 A \_h a p_{-} l a$ & CCCT & 9.89 \\
\cline { 2 - 4 } & $7 A_{-} h a p_{-} l b$ & GT TC & $14.31^{* *}$ \\
\hline
\end{tabular}
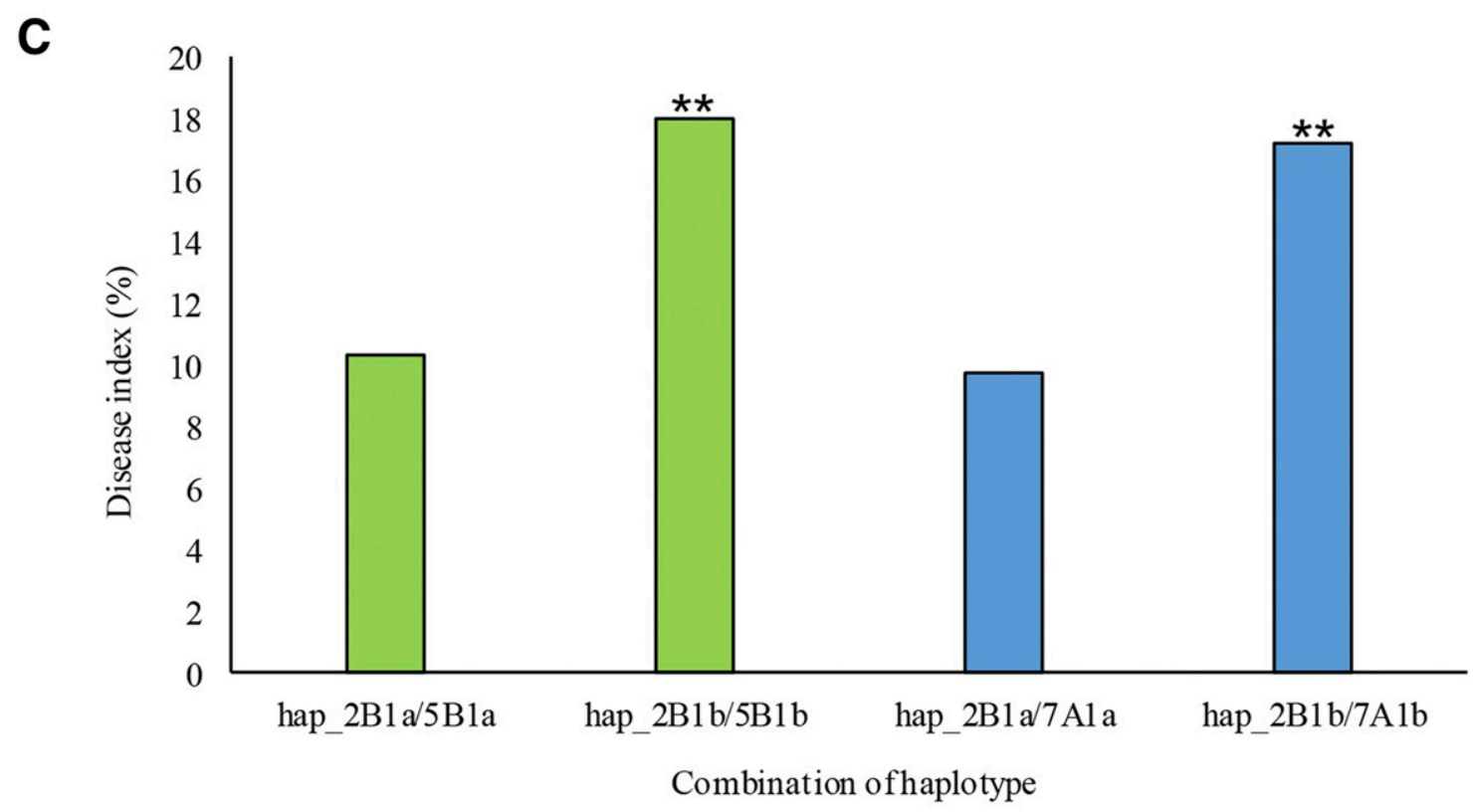

Fig. 4. Haplotype analysis of significant single-nucleotide polymorphism (SNPs) on chromosome $2 B, 5 B$, and 7A. A, Linkage disequilibrium heatmaps. B, Phenotypic comparison of cultivars with different haplotypes. C, Phenotypic comparison of pyramiding haplotypes. Asterisks ${ }^{* *}$ ) indicate $P<0.01$. 
STRUCTURE software 2.3.4 with unlinked markers $\left(r^{2}=0\right)$ (Pritchard et al. 2000). The number of subpopulations $(k)$ was implemented by a burn-in of 1,000 iterations followed by 1,000 Monte Carlo Markov Chain replicates in a putative range of 1 to 10. Linkage disequilibrium (LD) among markers was calculated for the A, B, and D genomes using the PLINK software. The window size for LD calculation was set based on the number of SNPs located in each genome. Pairwise LD was measured using the squared allele frequency correlations, according to Weir (Williams 1996), and assessed by calculating $r^{2}$ for pairs of SNP loci. GWAS was implemented using the mixed linear model $(\mathrm{PCA}+\mathrm{K})$ using the GAPIT package in $\mathrm{R}$ software (Zhang et al. 2010). The threshold for $P$ value was calculated using a modified

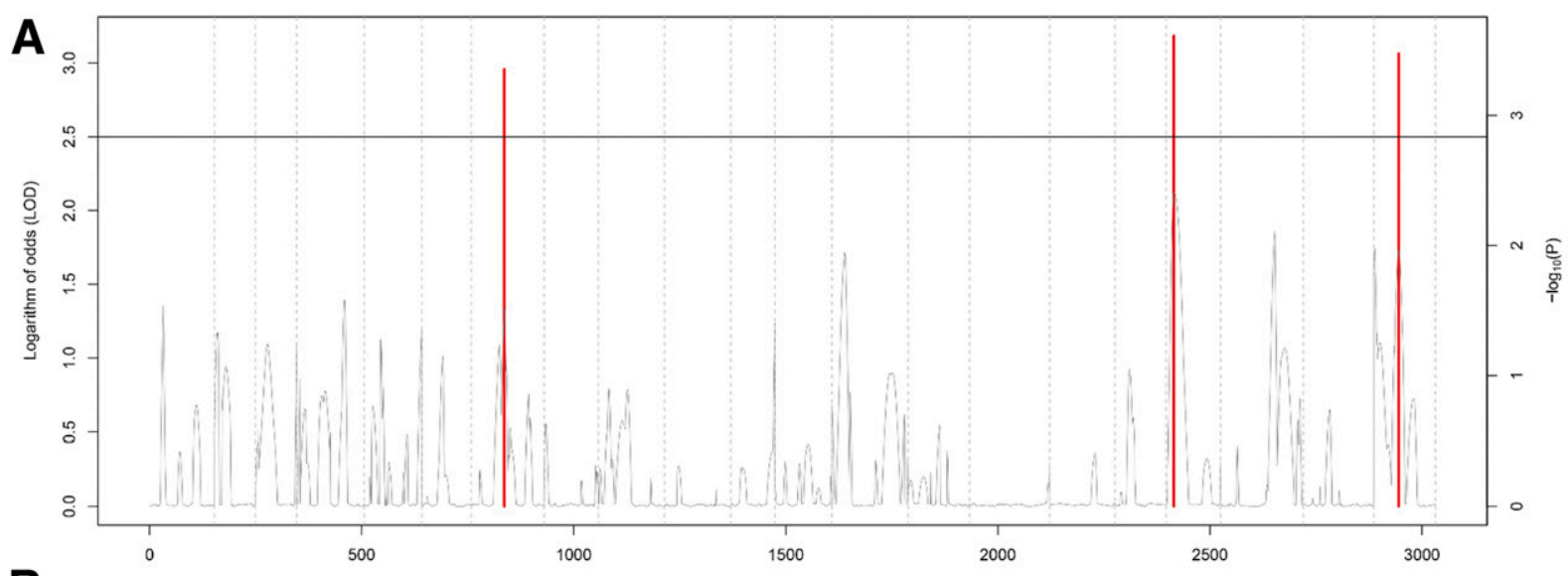

B

\begin{tabular}{lcccccccc}
\hline Name & Chr & $\begin{array}{c}\text { Additive } \\
\text { effect }\end{array}$ & LOD & $\begin{array}{c}\text { Left } \\
\text { marker }\end{array}$ & $\begin{array}{c}\text { Left marker position } \\
(\mathrm{cM})\end{array}$ & $\begin{array}{c}\text { Right } \\
\text { marker }\end{array}$ & $\begin{array}{c}\text { Right marker } \\
\text { position }(\mathrm{cM})\end{array}$ & $\mathrm{r}^{2}(\%)$ \\
\cline { 2 - 9 } QBP.hau-3A & 3A & -0.73 & 2.9566 & wms2 & 7.52 & rPt-9057 & 79.36 & 6.76 \\
QBP.hau-6D & 6D & 0.78 & 3.1836 & wPt-6661 & 16.26 & wPt-1695 & 57.61 & 7.79 \\
QBP.hau-7D & 7D & -0.83 & 3.0624 & wPt-1100 & 51.93 & wms295 & 67.92 & 8.84 \\
\hline
\end{tabular}

Fig. 5. Genome-wide composite interval mapping results of the UC1110 $\times$ PI610750 population. A, The quantitative trait loci (QTL) map and B, detailed information about three QTL. Chr = chromosome and $\mathrm{cM}=$ centimorgans.

Table 3. Comparison of significant single-nucleotide polymorphism (SNPs) in this and previous studies

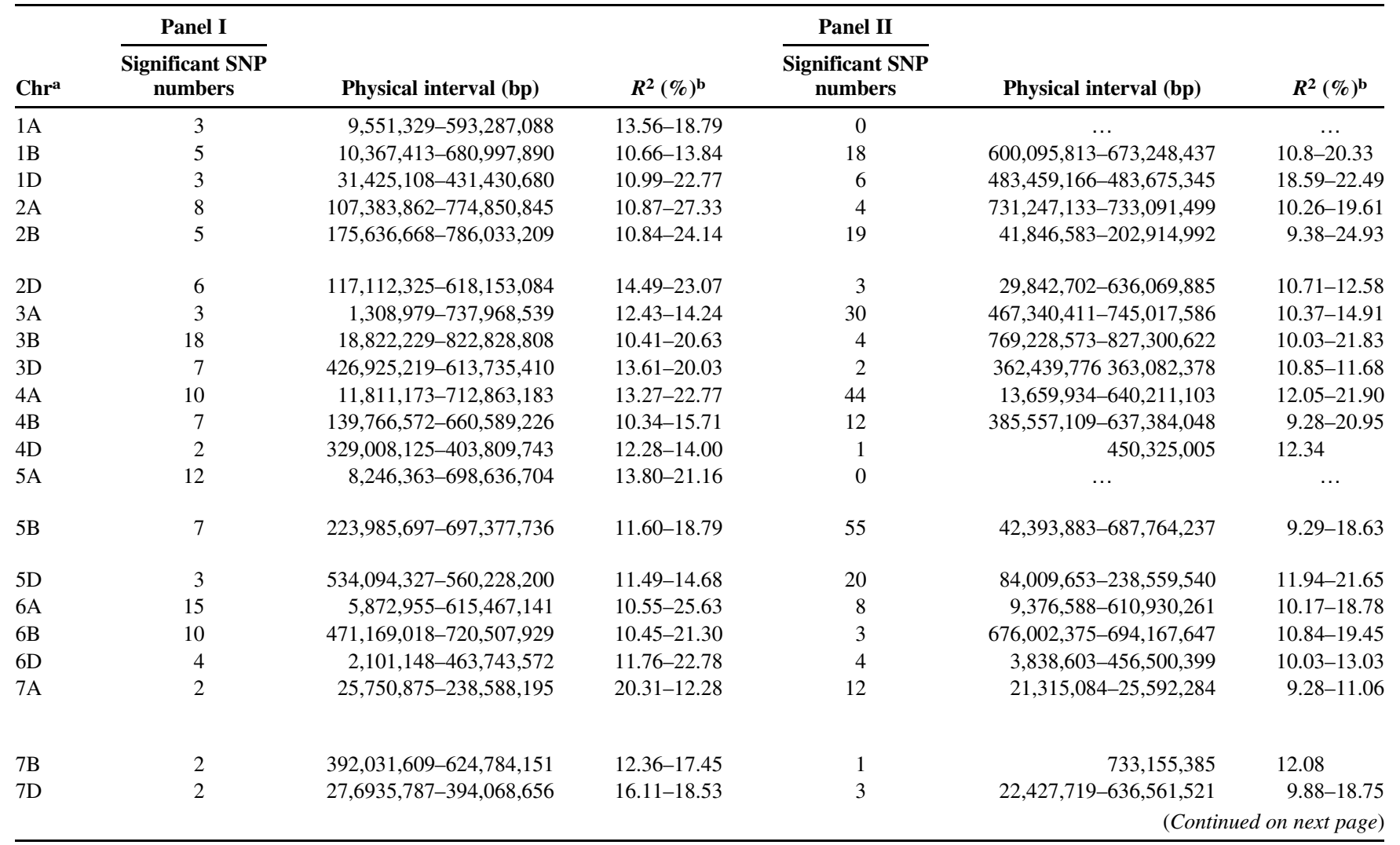

a Chromosome.

${ }^{\mathrm{b}} R^{2}$ of model with SNP.

${ }^{\mathrm{c}} \mathrm{QTL}=$ quantitative trait loci. 
Bonferroni correction (Genetic Type 1 Error Calculator, version 0.2). Haplotype analysis was performed using software Haploview 4.2.

QTL analysis and haplotype analysis. The genetic linkage map of the UP population previously constructed by Lowe et al. (2011) was composed of 1,494 polymorphic probes (SSRs, diversity arrays technologies, and expressed sequence tags), and 558 unique loci were used to detect QTL related to black point resistance through the genome-wide composite interval mapping (GCIM) method (Wang et al. 2016; Wen et al. 2018) using the software QTL.gCIMapping.GUI_1. Compared with the CIM and empirical Bayes methods, the GCIM method had a higher power in QTL detection, greater accuracy in QTL effect estimation, and stronger robustness under various backgrounds. The threshold of the logarithm of odds scores was set at 2.5.

\section{Results}

Resistance investigation of black point in Chinese common wheat. For the BLUP value across the different environments, the assessment of black point disease in Chinese common wheat showed a mean black point of 8.18 and $10.31 \%$ in panel I and panel II, respectively. The phenotyping results showed a wide range of black point incidences of 0 to $61 \%$ in panel I and 0 to $68 \%$ in panel II across the four environments (Fig. 1). Only four wheat accessions (Linkang 5027, Xinong 6028, Yanyou 361, and Zhengzhou 8761) had mean black point incidences of less than $1.5 \%$ in the two panels, indicating their big potential for use in wheat black point resistance breeding. Further analysis showed that there were 81,266, 57, and 2 accessions having mean black point incidences of 0.1 to $4.99,5$ to $14.9,15$ to 29.9 , and $>30 \%$, respectively. The data suggested that $14.5 \%$ of the tested Chinese wheat cultivars belong to the moderately susceptible or susceptible categories, and they should receive more attention for widely planting in black-point-endemic regions. Representative cultivars with different levels of black point resistance were shown in Table 1 .

GWAS. After filtering, 41,561 SNPs for the wheat 90K SNP array and 395,783 SNPs for the 660K SNP array were retained for GWAS analysis. In total, 136 and 250 SNPs were significant in panels I and II, respectively, across the four environments (Fig. 2), and they were mainly on chromosomes 3A, 4A, and 5B, with PVE of 9.29 to $24.93 \%$ (Supplementary Table S1). Of all significant SNPs, 27 were significant in multiple environments, and they were mainly on $2 \mathrm{~A}, 2 \mathrm{~B}, 3 \mathrm{~B}, 4 \mathrm{~A}, 6 \mathrm{~A}$, and $6 \mathrm{~B}$ (Table 2). A $t$-test analysis indicated that three SNPs (BS00097147_51, BS00087695_51, and Tdurum_contig11114_335) were significant in four environments $(P<0.01)$ and their mean PVE values were 10.84, 13.45 , and $20.63 \%$, respectively. Cultivars with alleles TT at BS00097147_51, GG at BS00087695_51, and AA at Tdurum_contig11114_335 showed significantly higher black point resistance and were regarded as superior alleles (Fig. 3). Pyramiding analysis of these three loci showed that cultivars with three superior and inferior alleles showed the averaged black point of 8.13 and $42.33 \%$, respectively.

Haplotype analysis (Fig. 4) indicated that the representative significant SNPs on 2B were clustered into the block $B P \_h a p \_2 B 1$, and the two haplotypes at this locus showed significant difference on black point $(9.99 \%$ for BP_hap_2Bla and $16.72 \%$ for BP_hap_2Blb). The significant SNPs on 5B formed the block $B P \_h a p \_5 B 1$, and cultivars with $B P \_h a p \_5 B 1 a$ showed a significantly lower black point incidence $(10.16 \%)$ than cultivars with BP_hap_5B1b $(12.97 \%)$. The significant SNPs on 7A formed the block BP_hap 7Al, and cultivars with $B P \_h a p \_7 A l a$ showed a significantly lower black point (9.89\%) than cultivars with $B P \_h a p \_7 A 1 b(14.31 \%)$. Blast of the significant SNPs in the genome database of Chinese Spring showed that these SNPs ranged from $42,179,859$ to $42,187,832 \mathrm{bp}$ on $2 \mathrm{~B}, 685,349,950$ to $685,392,702$ bp on $5 \mathrm{~B}$, and $24,296,895$ to $24,375,739 \mathrm{bp}$ on $7 \mathrm{~A}$. Pyramiding analysis indicated that cultivars with BP_hap_2B1a,BP_hap_5Bla, and BP_hap_7Ala showed a mean black point incidence of $9.67 \%$. There were no cultivars with the combination of $B P \_h a p \_2 B 1 b, B P \_h a p \_5 B 1 b$, and $B P \_h a p \_7 A 1 b$. Cultivars with $B P \_h a p \_2 B 1 a$ and $B P \_h a p \_5 B 1 a$ showed significantly lower black point (10\%) than cultivars with $B P \_h a p \_2 B 1 b$ and $B P$ $h a p \_5 B 1 b(18 \%)$, and those with BP_hap_2Bla and BP_hap_7Ala showed significantly lower black point $(9.7 \%)$ than those with $B P_{-}$ hap_2Blb and BP_hap_7Alb (17.2\%) (Fig. 4C).

Biparental QTL mapping for black point. Three QTL for black point resistance were detected in the UP population (Fig. 5), and

Table 3. (Continued from previous page)

\begin{tabular}{|c|c|c|}
\hline QTL intervalc & QTL, gene, markers & Intervals in Liu et al. (2017) \\
\hline$\ldots$ & $\ldots$ & $\ldots$ \\
\hline$\ldots$ & $\ldots$ & $\ldots$ \\
\hline$\ldots$ & & \\
\hline & IWB39123 (Liu et al. 2016) & $709,831,643-709,831,743 ; 712,188,721-712,187,200$ \\
\hline$\ldots$ & $\begin{array}{l}\text { abg356, wmc154, wmc149 (Lehmensiek et al. 2004); IWB516 (Liu } \\
\text { et al. 2016) }\end{array}$ & $714,389,068-714,388,998$ \\
\hline$\ldots$ & gwm484 (Lehmensiek et al. 2004) & $\ldots$ \\
\hline $60,200,972 \pm$ & IWA94 (Liu et al. 2016) & $9,605,904-9,605,974$ \\
\hline$\ldots$ & IWB633 (Liu et al. 2016) & $58,767,930-58,768,000 ; 695,967,481-695,967,411$ \\
\hline$\ldots$ & wmc375 (Lehmensiek et al. 2004) & $4,066,092-4,066,162$ \\
\hline$\ldots$ & germin (Lehmensiek et al. 2004) & $6,961,084-6,961,154 ; 504,944,902-504,944,832$ \\
\hline$\ldots$ & $\ldots$ & $\ldots$ \\
\hline$\ldots$ & $\ldots$ & $\ldots$ \\
\hline$\ldots$ & IWB13628 (Liu et al. 2016) & $\begin{array}{c}32,887,598-32,887,698 ; 535,780,381-535,780,311 \\
592,276,555-592,276,708\end{array}$ \\
\hline$\ldots$ & $\cdots$ & $\begin{array}{c}302,177,272-302,177,428 ; 531,539,253-531,539,323 ; \\
556,183,885-556,183,955\end{array}$ \\
\hline$\ldots$ & $\ldots$ & $\ldots$ \\
\hline$\ldots$ & IWB11102 (Liu et al. 2016) & $94,211,460-94,211,390$ \\
\hline$\ldots$ & $\ldots$ & $676,210,414-676,210,344$ \\
\hline $5,806,474-47,062,712$ & $\ldots$ & $217,194,463-217,194,533$ \\
\hline$\ldots$ & $\begin{array}{c}\text { gwm60 (Lehmensiek et al. 2004); } \\
\text { IWB53563, } \\
\text { IWA6562 (Liu et al. 2016) }\end{array}$ & $\begin{array}{c}88,862,791-88,862,721 ; 136,398,412-136,398,482 ; \\
609,508,901-609,508,971 ; 670,876,731-670,876,661 ; \\
708,211,110-708,211,040\end{array}$ \\
\hline$\ldots$ & IWA7589 (Lehmensiek et al. 2004) & $729,224,017-729,224,087$ \\
\hline $41,402,847-53,640,852$ & $\ldots$ & $129,917,622-129,917,692 ; 615,826,844-615,826,914$ \\
\hline
\end{tabular}


they were named QBP.hau-3A (approximately wms2 to rPt-9057), QBP.hau-6D (approximately wPt-6661 to wPt-1695), and QBP.hau$7 D$ (approximately $w P t-1100$ to $w m s 295$ ), with PVE of 6.76, 7.79, and $8.84 \%$, respectively. $Q B P . h a u-3 A$ and $Q B P . h a u-7 D$ were derived from UC1110 and QBP.hau-6D from PI610750. Further analysis of their physical positions in the Chinese Spring genome database showed that $Q B P . h a u-3 A$ was located at position $60,200,972 \mathrm{bp}$, $Q B P . h a u-6 D$ in a range between 5,806,474 and 470,627,654 bp, and QBP.hau-7D between 41,403,730 and 53,641,214 bp.

A combination of GWAS and QTL results showed that six significant SNPs (AX-109392024, GENE-3854_620, AX-94669779, AX94973555, Jagger_c5046_63, and GENE-4021_496) revealed by GWAS were within the interval of $Q B P . h a u-6 D$, suggesting that $Q B P$.hau-6D is an important novel QTL for resistance to black point because it was never reported before.

\section{Discussion}

Black point is a serious threat for wheat quality and production (Kahl et al. 2015). There was no absolute resistance in wheat cultivars and most cultivars would be easily affected by the disease under high moisture and other abiotic stresses (Mak et al. 2006; Sissons et al. 2010). Therefore, there is an urgent need to select resistant cultivars and find key genes for breeding new cultivars to control black point. Previously, Liu et al. (2017) used 166 accessions to evaluate for black point resistance and found that $86.1 \%$ (143) were susceptible (black point score $>10 \%$ ). Li et al. (2014) and El-Gremi et al. (2017) reported that the incidence of black point in wheat ranged from 0.3 to $66.7 \%$. In the present study, all wheat accessions tested showed black point incidence of 0 to $68 \%$ in multiple environments. There were some cultivars showing high resistance across four environments, such as Yumai8, Yanyou 361, Xiaoyan6, Zhengzhou 8761, and Xinong 6028, and they could be widely used in wheat black point resistance breeding. However, some cultivars (i.e., Yan 893, Xuan, and Yimai 8) showed high susceptibility to black point in all environment and should be avoided for planting in black-point-endemic areas. The present study illustrated the distribution of black point reactions in current popular wheat cultivars of the Huanghuai Valley, and could provide valuable information for utilization of wheat cultivars in this region and also provide useful resistant germplasm for wheat breeding programs concerning black point.

In the past few decades, most of the previous studies on wheat black point mainly focused on pathogen identification, biological characteristic, disease cycle, and management (Busman et al. 2012; Kahl et al. 2015; Somma et al. 2019; Williamson 1997), though some studies identified genetic loci related to black point resistance (Lehmensiek et al. 2004; Liu et al. 2016, 2017) (Table 3). Lehmensiek et al. (2004) detected six QTL related to black point resistance with PVE of 4 to $15 \%$ in the population of Sunco/Tasman, and three of them on 2A, 2D, and 7A explained 12 to $18 \%$ of phenotypic variance. Liu et al. (2016) found nine QTL for black point resistance in an RIL population of Linmai2/Zhong 892 on chromosomes $2 \mathrm{AL}, 2 \mathrm{BL}, 3 \mathrm{BL}$, $5 \mathrm{AS}, 6 \mathrm{~A}$, and 7AL. In the present study, we identified some important QTL for resistance to modulate wheat black point, and their physical positions overlapped with or covered previous QTL. However, three QTL (QBP.hau-3A, QBP.hau-6D, and QBP.hau-7D) that we identified in the RIL population have never been reported in previous studies, suggesting that they are possibly novel genetic loci for black point. Liu et al. (2017) found that the resistant SNPs were mainly located on 2A, 2B, 3A, 3B (2), 3D, 4B (2), 5A (3), 5B (3), $6 \mathrm{~A}, 6 \mathrm{~B}, 6 \mathrm{D}, 7 \mathrm{~A}(5), 7 \mathrm{~B}$, and 7D (2) by GWAS, with PVE of 7.9 to $18.0 \%$. Compared with this study, we found that some overlapped intervals were on $2 \mathrm{~A}, 2 \mathrm{~B}, 3 \mathrm{~A}, 3 \mathrm{~B}, 4 \mathrm{~A}, 5 \mathrm{~A}, 5 \mathrm{~B}, 6 \mathrm{~A}, 6 \mathrm{~B}, 6 \mathrm{D}$, and $7 \mathrm{D}$, indicating that these intervals possibly have important genetic loci related to black point.

In the present study, significant SNPs related to black point were mainly distributed on $2 \mathrm{~B}, 5 \mathrm{~B}$, and $7 \mathrm{~A}$. Haplotype analysis indicated that the cultivars with superior haplotype $B P \_h a p \_2 B 1 a, B P \_h a p_{-}$ $5 B 1 a$, or $B P \_h a p \_7 A 1 a$ showed significantly higher resistance to black point than cultivars with inferior haplotype $B P \_h a p \_2 B 1 b$, $B P \_h a p \_5 B 1 b$, or $B P \_h a p \_7 A 1 b$. Pyramiding analysis indicated that
192 cultivars such as Xinyanmai 98, Shenzhou 209, Kaimai 27, and Jinmai 109 with the superior haplotypic combination $B P \_h a p \_2 B 1 a$, BP_hap_5Bla, and BP_hap_7Ala showed a mean black point incidence of $9.67 \%$, and they could be considered as germplasms resistant to black point for use in wheat breeding programs.

Previous studies showed that QTL resistant to black point were mainly mapped to $2 \mathrm{~A}, 2 \mathrm{D}, 7 \mathrm{~A} 2 \mathrm{AL}, 2 \mathrm{BL}, 3 \mathrm{BL}, 5 \mathrm{AS}, 6 \mathrm{~A}$, and 7AL in different RIL populations (Lehmensiek et al. 2004, Liu et al. 2016). In the present study, QBP.hau-3A, QBP.hau-6D, and $Q B P . h a u-7 D$ were possibly novel genetic loci to control black point resistance because they were not previously reported in biparental populations, though a number of significant SNPs were within the intervals of these three QTL in some GWAS results using germplasm panels (Lehmensiek et al. 2004; Liu et al. 2016, 2017). We also found six significant SNPs within the $Q B P$.hau- $6 D$ region in this study. Therefore, QBP.hau-6D should be used as a superior QTL for marker-assisted selection to improve wheat black point resistance. In addition, pyramiding several QTL conferring black point resistance is possibly more effective to improve black point resistance in wheat breeding programs.

\section{Literature Cited}

Busman, M., Desjardins, A. E., and Proctor, R. H. 2012. Analysis of fumonisin contamination and the presence of Fusarium in wheat with kernel black point disease in the United States. Food Addit. Contam. 29:1092-1100.

Chen, J., Zhang, F., Zhao, C., Lv, G., Sun, C., Pan, Y., Guo, X., and Chen, F. 2019 Genome-wide association study of six quality traits reveals the association of the TaRPP13L1 gene with flour colour in Chinese bread wheat. Plant Biotechnol. J. 17:2106-2122.

Conner, R. L., and Davidson, J. G. N. 1988. Resistance in wheat to black point caused by Alternaria alternata and Cochliobolus sativus. Can. J. Plant Pathol. 68:351-359.

Cromey, M. G., and Mulholland, R. I. 1988. Black point of wheat: Fungal associations, cultivar susceptibility, and effects on grain weight and germination. N. Z. J. Agric. Res. 31:51-56.

Desjardins, A. E., Busman, M., Proctor, R. H., and Stessman, R. 2007. Wheat kernel black point and fumonisin contamination by Fusarium proliferatum. Food Addit. Contam. 24:1131-1137.

Dexter, J. E., and Matsuo, R. R. 1982. Effect of smudge and black point, mildewed kernels, and ergot on durum wheat quality. Cereal Chem. 59:63-69.

El-Gremi, S. M., Draz, I. S., and Youssef, W. A. 2017. Biological control of pathogens associated with kernel black point disease of wheat. Crop Prot. 91: 13-19.

Ellis, S. A., Gooding, M. J., and Thompson, A. J. 1996. Factors influencing the relative susceptibility of wheat cultivars (Triticum aestivum L.) to black point. Crop Prot. 15:69-76.

Fernandez, M. R., and Conner, R. L. 2011. Black point and smudge in wheat. Prairie Soils Crops 4:158-164

Fernandez, M. R., Wang, H., and Singh, A. K. 2014. Impact of seed discoloration on emergence and early plant growth of durum wheat at different soil gravimetric water contents. Can. J. Plant Pathol. 36:509-516.

Fuerst, E. P., Okubara, P. A., Anderson, J. V., and Morris, C. F. 2014. Polyphenol oxidase as a biochemical seed defense mechanism. Front. Plant Sci. 5:689.

Hudec, K. 2007. Pathogenicity of fungi associated with wheat and barley seedling emergence and fungicide efficacy of seed treatment. Biologia 62:287-291.

Kahl, S. M., Ulrich, A., Kirichenko, A. A., and Müller, M. E. H. 2015. Phenotypic and phylogenetic segregation of Alternaria infectoria from small-spored Alternaria species isolated from wheat in Germany and Russia. J. Appl. Microbiol. 119:1637-1650.

Kang, Y., Zhang, Y., Li, H., and Guo, Y. 1999. Research status of wheat black point disease in China. Acta Tritical Crops 19:58-60.

Kumar, J., Schäfer, P., Hückelhoven, R., Langen, G., Baltruschat, H., Stein, E., Nagarajan, S., and Kogel, K. H. 2002. Bipolaris sorokiniana, a cereal pathogen of global concern: Cytological and molecular approaches towards better control. Mol. Plant Pathol. 3:185-195.

Lehmensiek, A., Campbell, A. W., Sutherland, M. W., Williamson, P. M., and Daggard, G. E. 2004. QTLs for black-point resistance in wheat and the identification of potential markers for use in breeding programs. Plant Breed. 123:410-416

Li, Q., Qin, Z., Jiang, Y., Shen, C., Duan, Z., and Niu, J. S. 2014. Screening wheat genotypes for resistance to black point and the effects of diseased kernels on seed germination. J. Plant Dis. Prot. 121:79-88.

Liu, J., He, Z., Rasheed, A., Wen, W., Yan, J., Zhang, P., Wan, Y., Zhang, Y., Xie, C., and Xia, X. 2017. Genome-wide association mapping of black point reaction in common wheat (Triticum aestivum L.). BMC Plant Biol. 17:220.

Liu, J., He, Z., Wu, L., Bai, B., Wen, W., Xie, C., and Xia, X. 2016. Genome-wide linkage mapping of QTL for black point reaction in bread wheat (Triticum aestivum L.). Theor. Appl. Genet. 129:2179-2190. 
Lowe, I., Jankuloski, L., Chao, S., Chen, X., See, D., and Dubcovsky, J. 2011. Mapping and validation of QTL which confer partial resistance to broadly virulent post-2000 North American races of stripe rust in hexaploid wheat. Theor. Appl. Genet. 123:143-157.

Mak, Y., Willows, R. D., Roberts, T. H., Wriqley, C. W., Sharp, P. J., and Copeland, L. 2006. Black point is associated with reduced levels of stress, disease- and defence-related proteins in wheat grain. Mol. Plant Pathol. 7: 177-189.

Maloy, O. C., and Specht, K. L. 1988. Black point of irrigated wheat in Central Washington. Plant Dis. 72:1031-1033.

Marasas, W. F. 2001. Discovery and occurrence of the fumonisins: A historical perspective. Environ. Health Perspect. 109:239-243.

March, T. J., Able, J. A., Schultz, C. J., and Able, A. J. 2007. A novel late embryogenesis abundant protein and peroxidase associated with black point in barley grains. Proteomics 7:3800-3808

Palacios, S. A., Susca, A., Haidukowski, M., Stea, G., Cendoya, E., Ramírez, M. L., Chulze, S. N., Farnochi, M. C., Moretti, A., and Torres, A. M. 2015. Genetic variability and fumonisin production by Fusarium proliferatum isolated from durum wheat grains in Argentina. Int. J. Food Microbiol. 201: $35-41$.

Perelló, A., Moreno, M., and Sisterna, M. 2008. Alternaria infectoria speciesgroup associated with black point of wheat in Argentina. Plant Pathol. 57:379.

Porta, H., and Rocha-Sosa, M. 2002. Plant lipoxygenases, physiological and molecular features. Plant Physiol. 130:15-21.

Pritchard, J. K., Stephens, M. J., and Donnelly, P. J. 2000. Inference of population structure using multilocus genotype data. Genetics 155:945-959.

Purcell, S., Neale, B., Todd-Brown, K., Thomas, L., Ferreira, M. A. R., Bender, D., Maller, J., Sklar, P., Bakker, W. I. P., Daly, M. J., and Sham, P. C. 2007. Plink: A tool set for whole-genome association and population-based linkage analyses. Am. J. Hum. Genet. 81:559-575.

Rees, R. G., Martin, D. J., and Law, D. P. 1984. Black point in bread wheat. Effects on quality and germination and fungal associations. Aust. J. Exp. Agric. 24: 601-605.

Sissons, M., Sissons, S., and Egan, N. 2010. The black point status of selected tetraploid species and Australian durum wheat and breeding lines. Crop Sci. 50:1279-1286.
Somma, S., Amatulli, M. T., Masiello, M., Moretti, A., and Logrieco, A. F. 2019. Alternaria species associated to wheat black point identified through a multilocus sequence approach. Int. J. Food Microbiol. 293:34-43.

Southwell, R. J., Brown, J. F., and Wong, P. T. W. 1980. Effect of inoculum density, stage of plant growth and dew period on the incidence of black point caused by Alternaria alternata in durum wheat. Ann. Appl. Biol. 96:29-35.

Sun, C., Zhang, F., Yan, X., Zhang, X., Dong, Z., Cui, D., and Chen, F. 2017. Genome-wide association study for 13 agronomic traits reveals distribution of superior alleles in bread wheat from the Yellow and Huai Valley of China. Plant Biotechnol. J. 15:953-969.

Tomás-Barberán, F. A., and Espín, J. C. 2001. Phenolic compounds and related enzymes as determinants of quality in fruits and vegetables. J. Sci. Food Agric. 81:853-876.

Walker, K. R., Able, J. A., Mather, D. E., and Able, A. J. 2008. Black point formation in barley: Environmental influences and quantitative trait loci. Aust. J. Agric. Res. 59:1021-1029.

Wang, S., Wen, Y., Ren, W., Ni, Y., Zhang, J., Feng, J., and Zhang, Y. 2016. Mapping small-effect and linked quantitative trait loci for complex traits in backcross or DH populations via a multi-locus GWAS methodology. Sci Rep. 6:29951.

Wen, Y., Zhang, Y., Zhang, J., Feng, J., Jim, M. D., and Zhang, Y. 2018. An efficient multi-locus mixed model framework for the detection of small and linked QTLs in $\mathrm{F}_{2}$. Brief. Bioinf. 20:1-12.

Williams, J. T. 1996. Genetic data analysis II: Methods for discrete population genetic data by Bruce S. Weir. Hum. Biol. 4:583-586.

Williamson, P. M. 1997. Black point of wheat: In vitro production of symptoms, enzymes involved, and association with Alternaria alternata. Aust. J. Agric. Res. 48:13-19.

Yang, X., Pan, Y., Singh, P. K., He, X., Ren, Y., Zhao, L., Zhang, N., Cheng, S., and Chen, F. 2019. Investigation and genome-wide association study for Fusarium crown rot resistance in Chinese common wheat. BMC Plant Biol. 19:153.

Zhang, Z., Ersoz, E., Lai, C.-Q., Todhunter, R. J., Tiwari, H. K., Gore, M. A., Bradbury, P. J., Yu, J., Arnett, D. K., Ordovas, J. M., and Buckler, E. S. 2010. Mixed linear model approach adapted for genome-wide association studies. Nat. Genet. 42:355-360. 